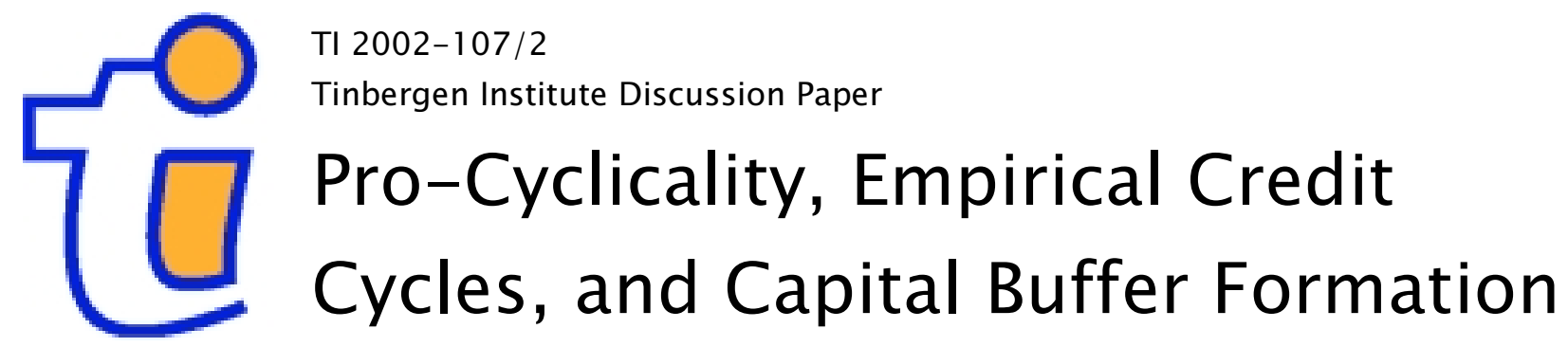

Siem Jan Koopman ${ }^{1,2}$

André Lucasl,2

Pieter Klaassen ${ }^{1,3}$

1 Faculty of Economics and Business Administration, Vrije Universiteit Amsterdam, 2 Tinbergen Institute, ${ }^{3} A B N-A M R O$ Bank NV, Amsterdam. 
Tinbergen Institute

The Tinbergen Institute is the institute for economic research of the Erasmus Universiteit Rotterdam, Universiteit van Amsterdam and

Vrije Universiteit Amsterdam.

Tinbergen I nstitute Amsterdam

Keizersgracht 482

1017 EG Amsterdam

The Netherlands

Tel.: +31.(0)20.5513500

Fax: $\quad+31 .(0) 20.5513555$

Tinbergen Institute Rotterdam

Burg. Oudlaan 50

3062 PA Rotterdam

The Netherlands

Tel.: $\quad+31 .(0) 10.4088900$

Fax: $\quad+31 .(0) 10.4089031$

Most TI discussion papers can be downloaded at

http://www.tinbergen.nl 


\section{Pro-Cyclicality, Empirical Credit Cycles, and Capital Buffer Formation}

First version: March 1, 2002

This version: October 15, 2002

Siem Jan Koopman ${ }^{\S}$, André Lucas ${ }^{\ddagger \S}$, Pieter Klaassen $^{\dagger \ddagger}$

$\S_{\text {}}$ Tinbergen Institute Amsterdam, Keizersgracht 482, NL-1017 EG Amsterdam, the Netherlands

IDepartment of Econometrics, Vrije Universiteit Amsterdam, De Boelelaan 1105, NL-1081 HV Amsterdam, The Netherlands

${ }^{\dagger}$ ABN-AMRO Bank NV, P.O. Box 283, NL-1000 EA Amsterdam, The Netherlands

${ }_{\ddagger}^{\ddagger}$ Department of Finance and Financial Sector Management, Vrije Universiteit Amsterdam, De Boelelaan 1105, NL- 1081 HV Amsterdam, The Netherlands 


\title{
Pro-Cyclicality, Empirical Credit Cycles and Capital Buffer Formation
}

\author{
First version: March 1, 2002 \\ This version: October 15, 2002
}

\begin{abstract}
We model 1927-1997 U.S. business failure rates using a time series approach based on unobserved components. Clear evidence is found of cyclical behavior in default rates. The cycle has a period of around 10 years. We also detect longer term movements in default probabilities and default correlations. Our findings have important implications for portfolio credit risk analysis. First, a static analysis of portfolio credit risk can underestimate credit risk significantly by not accounting for the dynamic and cyclical behaviour of default probabilities. Second, estimating default correlations over long horizons without accounting for time variation may lead to misspecified risk management models. We highlight the main effects in an actual credit risk experiment, addressing the issue of pro-cyclicality in ratings and capital buffer formation. It turns out that dynamic models anticipate much better on required capital buffer increases than rating strategies based on recent historical data. In this way, dynamic credit risk models may help to alleviate part of the pro-cyclicality problem.
\end{abstract}

Key words: credit risk; ; pro-cyclicality; capital requirements; dynamic models; common factors; credit cycles; time varying parameters.

JEL Codes: G21; C19.

\section{Introduction}

Credit risk aspects for both corporates and financial institutions appear increasingly important. The year 2002 appears to become a record high with regards to the number of defaults and amount of unrecovered debt. The importance of credit risk has led to a recent surge in interest for credit risk modeling. This has resulted in several interesting lines of research. Both professional and academic papers appear in the areas of predicting bankruptcies, pricing credit risky instruments, measuring credit risk for portfolios, managing credit risk, and regulatory aspects of credit risk, including the issue of pro-cyclicality. For an overview, see Caouette, Altman, and Narayanan (1998), Basle Committee on Bank Supervision (2001), and Allen and Saunders (2002). In brief, the pro-cyclicality issue concerns the question whether risk sensitive capital requirements and contemporary methods for credit risk measurement cause an amplification of cyclical movements in the economy.

In this paper, we address the issues of credit risk measurement and pro-cyclicality. We use new data and a new modeling framework. We add to the current literature in three different ways. First, we focus on the time series dimension of data rather than the cross-section dimension to extract information on possible cyclical movements from the data. Most other studies, by contrast, use cross-sectional or panel data, see Nickell, Perraudin, and Varotto (2000), Kavvathas (2001), Carpenter, Whitesell, and Zakrajšek (2001), Bangia, Diebold, Kronimus, Schagen, and Schuermann (2002), Laeven and Majnoni (2002), Das, Freed, Geng, and Kapadia (2002), Allen and Saunders (2002). We use U.S. business failure rates over the period 


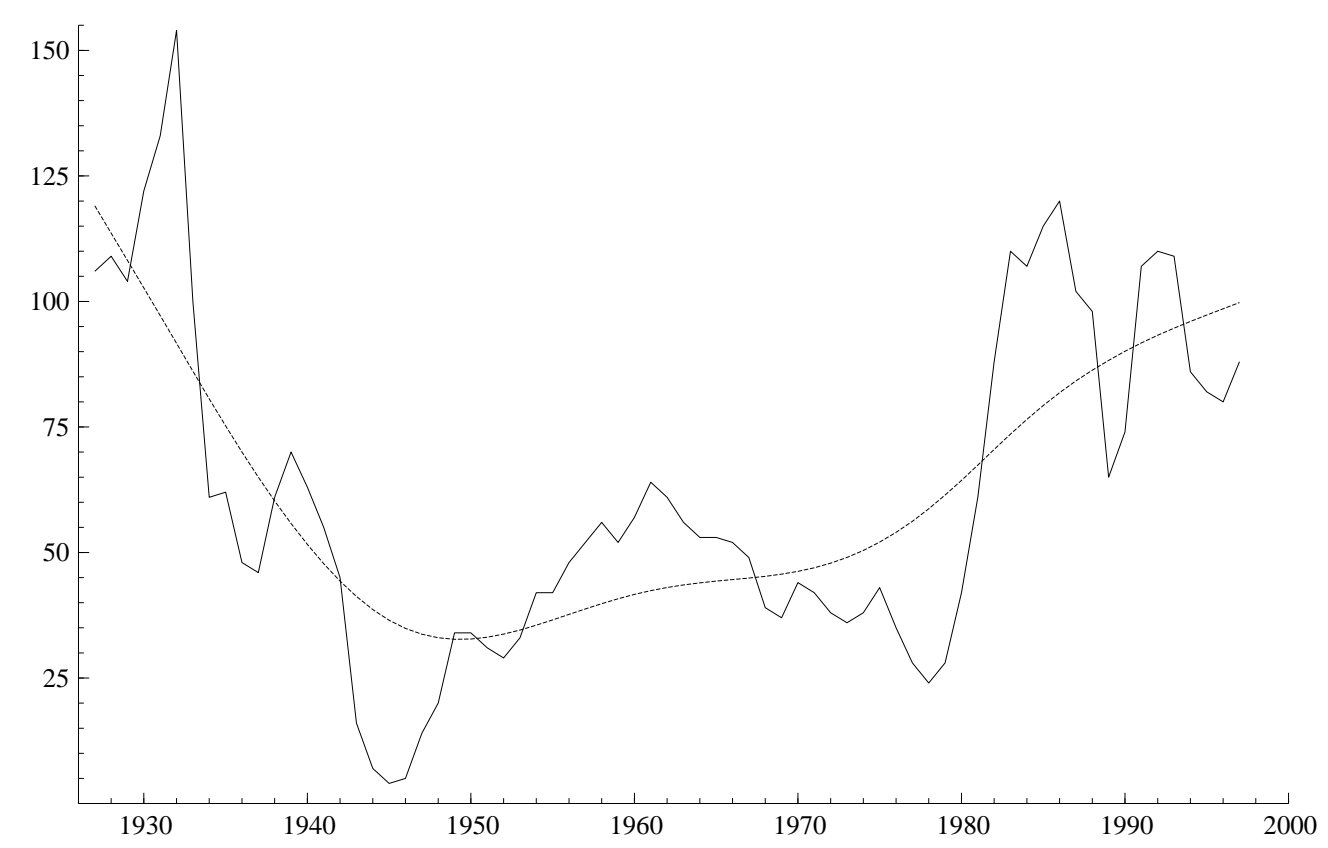

Figure 1: U.S. Business Failure Rates

Data taken from Dun and Bradstreet (1998). The figure also presents the HodrickPrescott trend for the data.

1927-1997 as visualized in Figure 1. The advantage of using time series data over a longer period is that more information can be extracted on the dynamics of business failures. Modeling the dynamics is more difficult for data which have a shorter time span, such as most of the cross-section and panel data used in earlier work. The fact that panel data tries to compensate the short time span by an enlarged cross-sectional dimension does not solve the difficulties with modeling the dynamic structure of business failures, see also Gordy and Heitfield (2002). Naturally, the use of a long time series may jeopardize the stability of the model and its parameters in time. We pay substantial attention to this in our econometric analysis.

The second contribution of our paper lies in the modeling framework used. We consider time series models based on unobserved components, see Harvey (1989), that aim at decomposing the business failure series into a stationary autoregressive component and a time-varying cyclical component. These components have a clear interpretation in the current credit risk context as systematic risk factors. The literature so far has largely focused on observable conditioning variables to model systematic risk and to link credit cycles to stages of the business cycle. Nickell, Perraudin, and Varotto (2000) use observed GDP growth to classify individual quarters as high, medium, or low-growth periods. They compute separate default and rating transition probabilities for each of these regimes. A similar approach is followed by Bangia, Diebold, Kronimus, Schagen, and Schuermann (2002), where NBER classifications of months as recessions and expansions are used. Kavvathas (2001) uses a duration model for rating transitions and incorporates macroeconomic variables such as the slope of the term structure to capture systematic effects on transition probabilities. All these studies show that systematic time-variation in default rates is present and that such time-variation may to some extent be captured by macroeconomic variables. There are two main drawbacks of an approach based on using observables as a proxy for systematic risk factors. First, there is little theory as to which observables would be optimal or even correct as a proxy for systematic risk. Though the informal argument in favor of using business cycle indicators to model credit cycles is well 
known, a casual inspection of Figure 1 reveals that business cycle peaks and troughs during the 50 s and 60s were not accompanied by corresponding pronounced movements in failure rates. Second, the use of observable conditioning variables implies a two-stage approach for credit risk management at the portfolio level. A typical credit risk analysis is built upon a model or set of scenarios for the default rate. Scenarios of future default rates require a model to generate scenarios for the conditioning variables, such as GDP or the term structure of interest rates. Models for the conditioning variables are usually estimated independently of the observed default data. In our latent variable framework, by contrast, we use an integrated approach and model the dynamics of the (latent) systematic risk component directly. This allows us to disentangle more gradual, long-term shifts in default probabilities from basic credit cycle patterns. It also helps us to focus on the dynamics of defaults directly, rather than indirectly via studying the dynamics of default related time series.

As a third contribution, our paper shows that the use of dynamic default rate models alleviates part of the pro-cyclicality problems. One of the worries of regulators is that during an upswing of the economy, banks will lower their capital levels. Such a decrease in capital may be spurred by risk sensitive capital requirements based on recent estimates of default probabilities. The use of estimated default probabilities based on banks' internal models is explicitly allowed in the new Basle Capital Accord, see Basle Committee on Bank Supervision (2001). As a result, capital levels may be too low at the peak of the cycle to cope with the subsequent downswing. The capital accumulation during the downswing may also be too slow. Moreover, the increases in capital may result in a credit crunch and thus worsen already adverse economic conditions, see Laeven and Majnoni (2002). To study the pro-cyclicality issue, we set up a simulation experiment for a stylized bank's balance sheet. We confront the implications of our dynamic models with an approach that mimics banks' current practice. The latter approach calibrates default probabilities and correlations to the recent past ( 5 and 10 years, respectively). It turns out that in our experiments the dynamic models result in a much more timely capital accumulation, especially during late $70 \mathrm{~s}-80 \mathrm{~s}$.

Our economic model is a reduced form credit risk model based on normally distributed risk factors. Though it resembles the CreditMetrics model in appearance, see Gupton, Finger, and Bhatia (1997), it is not a structural model. The difference can be noted by comparing our specification with some of the alternative dynamic extensions of the CreditMetrics model put forward in Finger (2000). The assumption of normality allows us to use the extensive literature and methodology for linear state space models, see, for example, Durbin and Koopman (2001). Alternative distributional specifications like those in Credit Suisse (1997) are much more difficult to handle in such a context. A difference between our model and other reduced form models such as Jarrow and Turnbull (1995), Jarrow, Lando, and Turnbull (1997), or especially Duffie and Singleton (1999), is that we model the default hazard rates implicitly rather than explicitly. As a consequence in our discrete time setting, the dynamics of the hazard rates are nontrivial. The converse holds for the default probability.

Using our latent modeling approach, we find three main empirical results. First, there is clear evidence of a stochastic cycle with a period between 9.5 and 11.5 years. This cycle can be interpreted as a credit cycle. Its period is roughly similar to familiar business cycle periods. Second, apart from this cycle, we also find more gradual dynamic behavior in default rates. Given the limited number of data points, there are two competing model specifications that fit the data equally well. The first model allows for second order dynamics in the long-term behavior of default rates. The second model has a second, long-term cycle with a period of about 40 years. As these models are empirically very close, we proceed by investigating whether they also yield similar implications with respect to dynamic capital requirements. Finally, we find significant evidence of volatility shifts in the cyclical component of default rates. This, in 
turn, indicates that over longer periods of time default correlations change. In particular, we find evidence of three different regimes for default correlations, namely up to the 40's, during the 50's up to a large part of the 70's, and from the late 70's onwards. Correlations were highest during the first period, more than halved during the second, and subsequently slightly increased during the third period.

The rest of the paper is set up as follows. Section 2 discusses the data and the credit risk model. Section 3 gives a brief exposition of unobserved components (or structural) time series models and their relation to our credit risk model. Section 4 contains the empirical results. Section 5 discusses the implications of our models for capital requirements, while Section 6 addresses the issue of pro-cyclicality. Section 7 concludes.

\section{Data and modeling approach}

The data used in this paper are U.S. failure rates from Dun and Bradstreet (1998). The data contain business failure frequencies for the United States over the period 1927-1997, see Figure 1. The numbers indicate businesses that ceased operations after assignment or bankruptcy; ceased operations with losses to creditors after such actions as foreclosure or attachment; voluntarily withdrew leaving unpaid debts; were involved in court actions such as receivership, reorganization or arrangement; or voluntarily compromised with creditors. This time series shows peaks in the early 1930's, the mid 1980's and the early 1990's. The series illustrates some of the well-known stages and developments in the US economy. Standard practice in empirical macroeconomic time series is to use the Hodrick-Prescott filter to extract a trend from the time series. This trend is also presented in Figure 1. By concentrating on the deviations from the Hodrick-Prescott trend, we obtain some first indications that the time series may be decomposed into a trend (or slowly varying) component and a cycle. However, this particular ("deterministic") decomposition is not optimal and rather restrictive. We adopt the arguments of Harvey and Jaeger (1993) and take a model-based approach as discussed further below and in the next section.

Given the data, we consider the following modeling framework. Let $S_{j t}$ be an unobserved surplus variable for firm $j$ at time $t$. If this variable drops below a critical threshold $c_{t}$, the firm defaults. Default can thus be modeled as a Bernoulli process with probability $\mathrm{P}\left[S_{j t}<c_{t}\right]$. This closely follows the set-up of for example CreditMetrics, one of the prominent models in credit risk management, see Gupton, Finger, and Bhatia (1997). It also bears close resemblance to CreditRisk $^{+}$(Credit Suisse (1997)), where the Bernoulli distribution is replaced by a Poisson approximation, see also Gordy (2000).

In order to allow for defaults to be correlated, we decompose $S_{j t}$ into a systematic and firm-specific risk factor,

$$
S_{j t}=\rho_{t} f_{t}+\sqrt{1-\rho_{t}^{2}} \cdot e_{j t}, \quad 0 \leq \rho_{t} \leq 1,
$$

where $f_{t}$ is the systematic risk factor and $e_{j t}$ is the firm-specific risk factor. Both are assumed to be standard normally distributed. The firm-specific risk factors are assumed to be uncorrelated across firms. The parameter $\rho_{t}$ then determines the amount of correlation between the surplus variables of different firms at time $t$, and it thereby influences the correlation between default events of different firms. The closer $\rho_{t}$ approaches one, the more prominent the systematic risk vis-à-vis the firm-specific risk. Note that the parameterization in (1) is such that $S_{j t}$ is standard normally distributed as well. This normalization is needed to identify the model, as $S_{j t}$ is not observed directly. Also note that we abstract from firm heterogeneity, as neither 
$c_{t}$ nor $\rho_{t}$ depends on $j$. This seems a natural approach given the limited data set at hand. Otherwise, the model would be poorly identified as we only observe a single time series.

Our approach bears close resemblance to the exogenous default probability framework of Jarrow and Turnbull (1995) and Duffie and Singleton (1999), but less to the structural approach of Merton (1974). At first sight, the close resemblance of (1) to the CreditMetrics modeling approach might incorrectly lead one to identify it as a structural model, see also Finger (2000). The difference is easily seen if, as an example, $f_{t}$ is an i.i.d. process. In that case, conditional on the systematic risk factor $f_{t}$, the probability of default is $P\left[e_{j t}<\left(c_{t}-\rho_{t} f_{t}\right) / \sqrt{1-\rho_{t}^{2}} \mid f_{t}\right]$. A typical structural model would account for the fact that a rise in $S_{j t}$ in one period, due to either a high $f_{t}$ or a high $e_{j t}$, also gives a reduced probability of default in the next period. Here, by contrast, a higher $e_{j t}$ (with identical $f_{t}$ ) will not result in a lower default probability over the next period. The exogeneity of the default event and the independence of exposures conditional on the systematic factor $f_{t}$ cause the model to be similar in spirit to the CreditRisk ${ }^{+}$ framework, which is typically labeled a reduced form model. The main difference lies in the distributional assumptions made for the risk factors.

Our model for the aggregate time series of actual observed failure rates is based on model (1) for individual firms. Define

$$
F_{n t}=\frac{1}{n} \sum_{j=1}^{n} 1\left(S_{j t}<c_{t}\right),
$$

where $1(\cdot)$ is the indicator function. The distribution of $F_{n t}$ is a sum of correlated Bernoulli variables, which, in general, is difficult to work with. We therefore consider a simple approximation ${ }^{1}$ to $F_{n t}$, namely $F_{t}=\lim _{n \rightarrow \infty} F_{n t}$. Following Finger (1999) and Lucas, Klaassen, Spreij, and Straetmans (2001), this approximation should work well given the large number of firms underlying the aggregate failure rate series. We obtain

$$
\begin{aligned}
F_{t} & =\lim _{n \rightarrow \infty} \frac{1}{n} \sum_{j=1}^{n} 1\left(S_{j t}<c_{t}\right) \\
\stackrel{\text { a.s. }}{=} & \lim _{n \rightarrow \infty} \frac{1}{n} \sum_{j=1}^{n} \mathrm{P}\left[S_{j t}<c_{t} \mid f_{t}\right] \\
& =\lim _{n \rightarrow \infty} \frac{1}{n} \sum_{j=1}^{n} \Phi\left(\frac{c_{t}-\rho_{t} f_{t}}{\sqrt{1-\rho_{t}^{2}}}\right) \\
& =\Phi\left(\frac{c_{t}-\rho_{t} f_{t}}{\sqrt{1-\rho_{t}^{2}}}\right),
\end{aligned}
$$

where $\Phi(\cdot)$ denotes the standard normal cumulative distribution function. We have thus established a relationship between the aggregate default rate $F_{t}$, which we identify with the observed US failure rate as depicted in Figure 1, and the unobserved elements of our credit risk model $f_{t}, c_{t}$ and $\rho_{t}$. Note that the above expression does not explicitly contain firm-specific risk factors, as it is assumed that idiosyncratic risk has been diversified completely. However, the functional form of the mapping from $f_{t}$ to $F_{t}$ is dictated by the distributional assumptions for the firm-specific risk factors.

\footnotetext{
${ }^{1} \mathrm{~A}$ different approximation is to replace the Bernoulli by a Poisson with the same mean. This works for sufficiently small default probabilities and is closely linked to the Credit Suisse (1997) approach. This approach, however, is less easy to generalize to situations where we are not only interested in defaults but also in credit rating changes, or to situations with large default probabilities.
} 
As mentioned in the introduction, one approach to identify the unobserved elements of our credit risk model is to substitute observed macroeconomic variables for $f_{t}$. This is done in Nickell et al. (2000), Bangia et al. (2002), and Kavvathas (2001). The advantage of this approach is that (prior) information on these macroeconomic variables can be exploited in the forecasting stage. The main drawback of using observables is that their relation to the unobserved credit cycle has to be stable over time. Moreover, there is an increased possibility of model misspecification because incorrect or insufficient macroeconomic variables are substituted to proxy for $f_{t}$. Finally, using observed variables implies that one has to construct a separate forecasting model for $f_{t}$ in order to forecast failure rates beyond the sample period. Constructing such models may be difficult and makes credit risk analyses prone to additional model misspecification risk. Separation of the modeling processes for defaults and macroeconomic variables also leads to inefficiencies and possibly even inconsistencies in case of simultaneity. Special testing procedures should therefore be performed to check for this, see for example Kavvathas (2001).

To circumvent these difficulties, we follow a time series approach in which $F_{t}$ is modeled by unobserved components. This approach keeps the middle between the approach based on observed variables as described above, and a completely reduced form approach based on BoxJenkins ARIMA models, see for example Hamilton (1994). The unobserved components time series model has the advantage over reduced-form models that the various components can be interpreted in economic terms as we can relate them to $\rho_{t}$ and $c_{t}$. This is shown in the next section.

\section{An unobserved components time series model}

\subsection{Trend - cycle decompositions}

The unobserved components time series model is based on the classic principle that a time series can be decomposed into interpretable unobserved components such as a trend, seasonal, cycle and irregular component. For example, macroeconomic time series typically feature a long term trend with cyclical variations around this trend. Further they are often characterized by trends with different growth rates for different periods and by cycles with time-varying characteristics. An appropriate model for such time series is given by

$$
y_{t}=\mu_{t}+\psi_{t}+\varepsilon_{t}, \quad t=1, \ldots, n,
$$

where $y_{t}$ represents the actual time series and where $\mu_{t}, \psi_{t}$, and $\varepsilon_{t}$ are the level, cycle, and irregular time series components, respectively. The level component is time-varying, and possibly exhibits a trend. These components are unobserved and modeled by stochastic processes. For example, one may specify the level component by

$$
\mu_{t+1}=\mu_{t}+\xi_{t}, \quad \xi_{t} \sim \mathrm{N}\left(0, \sigma_{\xi}^{2}\right),
$$

for a random walk process. This reduces to a fixed level model if $\sigma_{\xi}^{2}=0$. Alternatively, we can specify the level component as a stationary process by

$$
\begin{aligned}
\mu_{1, t+1} & =\mu_{1 t} \\
\mu_{2, t+1} & =\phi \mu_{2 t}+\xi_{t}, \quad \xi_{t} \sim \mathrm{N}\left(0, \sigma_{\xi}^{2}\right) \\
\mu_{t+1} & =\mu_{1 t}+\mu_{2 t},
\end{aligned}
$$

for a level with autoregressive characteristics. Models of type (3) nest familiar trend extracting procedures like the Hodrick-Prescott filter; see Harvey and Jaeger (1993) for a discussion. 
Various specifications for the cycle component $\psi_{t}$ exist. For example, we may wish to generate a cycle by an $\mathrm{AR}(2)$ model with the coefficients selected in the complex range of solutions. To enforce this restriction, we represent the model in terms of a trigonometric specification, that is

$$
\left(\begin{array}{c}
\psi_{2, t+1} \\
\psi_{2, t+1}^{*}
\end{array}\right)=\phi\left[\begin{array}{cc}
\cos \lambda & \sin \lambda \\
-\sin \lambda & \cos \lambda
\end{array}\right]\left(\begin{array}{c}
\psi_{2 t} \\
\psi_{2 t}^{*}
\end{array}\right)+\left(\begin{array}{c}
\omega_{2 t} \\
\omega_{2 t}^{*}
\end{array}\right)
$$

with frequency $\lambda$ and persistence parameter $|\phi|<1$. The disturbances are serially and mutually uncorrelated and they are normally distributed with mean zero and variance matrix

$$
\operatorname{Var}\left(\begin{array}{c}
\omega_{t} \\
\omega_{t}^{*}
\end{array}\right)=\sigma_{\omega}^{2} I_{2}
$$

where $I_{k}$ is the $k \times k$ identity matrix. The disturbances $\omega_{t}$ and $\omega_{t}^{*}$ have a common variance $\sigma_{\omega}^{2}$. This stochastic cycle specification generates a stationary cyclical process with a period of $p=2 \pi / \lambda$.

The model is completed by taking the irregular component $\varepsilon_{t}$ as a normally distributed random variable with mean zero and variance $\sigma_{\varepsilon}^{2}$. The irregular and other disturbances associated with the various components are mutually uncorrelated, both contemporaneously and between different time periods. A full discussion of this class of time series models and their dynamic properties is given by Harvey (1989).

\subsection{Model implementation for default risk}

The unobserved components modeling approach introduced in the previous subsection is flexible, but has little direct economic content. Therefore, we introduce two specific variants of model (3) that link directly to the parameters of economic interest for default rates as in (2). To understand the model's design, define $y_{t}=\Phi^{-1}\left(F_{t}\right)$, with $\Phi^{-1}(\cdot)$ the inverse cumulative standard normal distribution function, and $F_{t}$ the observed default rate.

Given the limited number of data points, it is non-trivial to distinguish between some of the non-nested unobserved component models. Therefore, we take a pragmatic route and present the results for 3 of the most competitive models. ${ }^{2}$ The first model is an AR(1) plus cycle model and is given by

$$
\begin{aligned}
\mu_{t+1} & =\phi_{1} \mu_{t}+\sqrt{1-\phi_{1}^{2}} \xi_{t} \\
\left(\begin{array}{c}
\psi_{2, t+1} \\
\psi_{2, t+1}^{*}
\end{array}\right) & =\phi_{2}\left(\begin{array}{cc}
\cos \lambda_{2} & \sin \lambda_{2} \\
-\sin \lambda_{2} & \cos \lambda_{2}
\end{array}\right)\left(\begin{array}{c}
\psi_{2 t} \\
\psi_{2 t}^{*}
\end{array}\right)+\sqrt{1-\phi_{2}^{2}}\left(\begin{array}{c}
\omega_{2 t} \\
\omega_{2 t}^{*}
\end{array}\right), \\
y_{t} & =c \sqrt{1+a^{2}+b_{t}^{2}}+a \mu_{t}+b_{t} \psi_{2 t}
\end{aligned}
$$

with $\left(\xi_{t}, \omega_{t}, \omega_{t}^{*}\right)^{\prime} \sim \mathrm{N}\left(0, I_{3}\right)$. The second model is an $\operatorname{AR}(2)$ plus cycle and replaces (7) by

$$
\mu_{t+1}=\left(\phi_{1}+\phi_{3}\right) \mu_{t}-\phi_{1} \phi_{3} \mu_{t-1}+\sqrt{\frac{\left(1-\phi_{1} \phi_{3}\right)\left(1-\phi_{1}^{2}\right)\left(1-\phi_{3}^{2}\right)}{1+\phi_{1} \phi_{3}}} \xi_{t},
$$

which has unit variance and autoregressive roots $\phi_{1}$ and $\phi_{3}$, respectively. The final model has a double cycle and reads

$$
y_{t}=c \sqrt{1+a^{2}+b_{t}^{2}}+a \psi_{1 t}+b_{t} \psi_{2 t},
$$

\footnotetext{
${ }^{2}$ We have experimented with other empirical models, including non-stationary models, and models involving an irregular component. The irregular component, however, was estimated to be zero for all our models and is therefore omitted. The models we present here have the best interpretation and empirical performance.
} 
with $\psi_{2 t}$ as before, and $\psi_{1 t}$ as in (8), but with $\left(\phi_{2}, \lambda_{2}\right)$ replaced by $\left(\phi_{1}, \lambda_{1}\right)$. We impose $\lambda_{1}<\lambda_{2}$, such that $\psi_{1 t}$ has a longer period than $\psi_{2 t}$.

All models have in common that they decompose the transformed default rates into a deterministic and possibly time varying level component $c \sqrt{1+a^{2}+b_{t}^{2}}$, a slowly evolving stochastic component $\mu_{t}$ or $\psi_{1 t}$, and a (short) cyclical component $\psi_{2 t}$. The fact that the loading $b_{t}$ of the short cyclical component may be time varying stems from the empirical findings in the next section. that

Given $y_{t}=\Phi^{-1}\left(F_{t}\right)$ and the definition of $F_{t}$ in (2), we obtain for our first and second model

$$
\begin{aligned}
\rho_{t}^{2} & =\frac{a^{2}+b_{t}^{2}}{1+a^{2}+b_{t}^{2}}, \\
c_{t} & =c \\
f_{t} & =\frac{-a \mu_{t}-b_{t} \psi_{2 t}}{\sqrt{a^{2}+b_{t}^{2}}}, \\
d_{t} & =\frac{\Phi_{2}\left(c, c ; \rho_{t}^{2}\right)-\Phi(c)^{2}}{\Phi(c)[1-\Phi(c)]} .
\end{aligned}
$$

with $d_{t}$ the default correlation, and $\Phi_{2}\left(\cdot, \cdot ; \rho_{t}^{2}\right)$ the bivariate normal distribution function with correlation parameter $\rho_{t}^{2}$. For the third model, only the expression for $f_{t}$ changes to

$$
f_{t}=\frac{-a \psi_{1 t}-b_{t} \psi_{2 t}}{\sqrt{a^{2}+b_{t}^{2}}}
$$

In economic terms, the parameter $\Phi(c)$ can thus be interpreted as the long-term default probability. In (13) it is restricted to be time invariant. Alternatively, one could include the slowly varying component $\left(\mu_{t}\right.$ or $\left.\psi_{1 t}\right)$ into the default threshold $c_{t}$. This is only relevant from the perspective of model interpretation, and not for the credit risk analyses based on the model. We therefore stick to (12) through (14), as the interpretation of $c$ as a long-term unconditional default probability also seems to be most widely accepted in the literature.

Note that we allow for time varying asset $\left(\rho_{t}^{2}\right)$ and default $\left(d_{t}\right)$ correlations by time variation in the parameter $b_{t}$, the loading of the short cyclical component. For $b_{t} \equiv b$, we are back in the setting with constant asset (and default) correlations. We use the parameter $b_{t}$ to test for evidence of any changes in default correlations over longer samples. If this is the case, this adds yet another dimension to the debate on the difficulty of estimating such correlations reliably, see Gordy and Heitfield (2002).

\section{Empirical results}

We present estimation results for the models presented in the previous section. We estimate the models with (TV) and without (CV) time-variation in the loading $b_{t}$ for the short cycle $\psi_{2 t}$. Time variation is introduced by 3 time dummies with breaks at 1949 and 1976. These values were found by optimizing the loglikelihood value of the model over the break dates. We also present some sensitivity analyses with respect to the choice of break dates.

The unknown parameters in the AR(1) plus cycle model are the component loadings $a, b_{t}$, and $c$, together with the autoregressive coefficients $\phi_{1}$ and $\phi_{2}$ and the cycle frequency $\lambda_{2}$. For the $\operatorname{AR}(2)$ plus cycle model and the double cycle model, there is an additional parameter in the form of the second autoregressive root $\phi_{3}$ and the frequency of the long cycle $\lambda_{1}$, respectively. 
For estimation purposes, we transform the parameters to satisfy the restrictions $0<\phi_{1}, \phi_{2}<$ $1,-1<\phi_{3}<1$, and $\lambda_{1}, \lambda_{2}<\pi$. The transformed parameters will be collected in the parameter vector $\theta$, and estimated using maximum likelihood. Details on parameter estimation and model diagnostics can be found in Harvey (1989) and Durbin and Koopman (2001). ${ }^{3}$

\subsection{Results for constant (short) cycle loading $b_{t} \equiv b$}

The estimation results for the models with a constant short factor loading $b_{t} \equiv b$ are given in Table 1 in the columns headed with CV.

We first discuss the results for the model with $\mathrm{AR}(1)$ and cycle component. The parameters in Table 1 for this model (AR1;CV) indicate that there is statistically significant evidence of a cycle. The period of the cycle is about 9.5 years, which roughly corresponds to a typical business cycle period. The dampening factor $\phi_{2}$ is high, 0.947 , which indicates that the cyclical effect is persistent. Consequently, one can expect cycles to be important and present in credit risk simulations based on this model for horizons as long as 10 years $\left(0.947^{10} \approx 0.58 \gg 0\right)$. Also the $\mathrm{AR}(1)$ component is very persistent with a near unit root of 0.979 . The implied asset correlation is $10 \%$, which is economically significant. The confidence interval around this correlation, however, is large, which shows that it is difficult to obtain a reliable estimate of the asset correlation given the limited number of 71 data points. This is mainly caused by the huge confidence interval for the loading $a$ of the (slowly varying) AR(1) component. Through (12) this translates directly into a wide confidence band for $\rho^{2}$. Note that the short cyclical component only explains about $7 \%\left(\right.$ or $\left.b^{2} /\left(a^{2}+b^{2}\right)\right)$ of the time variation in default rates. So the bulk of variation is captured by longer term movements as captured by the AR(1) component. With respect to the diagnostics of the model, there is significant evidence of nonnormality $(\mathrm{N})$ for this model and some evidence of missed dynamics $(Q(15))$. A close inspection of the smoothed estimates of the $\operatorname{AR}(1)$ and cyclical component revealed that the variance of $\psi_{2 t}$ varies considerably over time. From the 50's to the 70's, the cycle is almost flat compared to the war and pre-war years and the years from the 70's onwards. This suggests to allow for a time-varying loading $b_{t}$ of the cyclical component. We consider this further in the next subsection.

We now turn to the AR(2) model (see the column labeled AR2;CV). Given the results of the $\mathrm{AR}(1)$ model, it is not surprising that there is no sign of missed dynamics (insignificant $Q(15)$ ) for the model with $\operatorname{AR}(2)$ component. The increase in likelihood value $(L)$ for the addition of only one parameter is statistically significant at the $1 \%$ level. However, there is still a strong indication of nonnormality $(\mathrm{N})$. The restriction of both $\operatorname{AR}(2)$ roots to be real in this model is not binding. The estimates indicate that the (short) cyclical component $\psi_{2 t}$ is nonstationary $\left(\phi_{2}=1\right)$. The period of the cycle, however, appears reasonable: around 8.5 years. The nonstationarity appears due to possible misspecification in the constant cycle loading $b$, see also the next subsection. The short cyclical component only captures a very small part $(6 \%)$ of the total time variation in default rates. The $\operatorname{AR}(2)$ roots $\phi_{1}$ and $\phi_{3}$ are smaller than the root $\left(\phi_{1}\right)$ for the AR(1) plus cycle model, meaning that the slowly varying component is less persistent. Also the asset correlation parameter $\rho^{2}$ is now somewhat lower: $5.7 \%$ rather than $10 \%$.

Finally, we consider the double cycle model with constant factor loading $b$, see the CYC;CV column in Table 1. The parameter estimates and diagnostics are very similar to the $\operatorname{AR}(2)$ plus cycle model. The main difference lies in the $\operatorname{AR}(2)$ versus the long cycle component. The long cycle has a period of about 38 years and a decay factor of $\phi_{1}=0.977$. The long

\footnotetext{
${ }^{3}$ The computations are implemented using Ox Professional 3.0, the object-oriented matrix software of Doornik (2001), and SsfPack 2.2, the Ox package for state space analysis by Koopman, Shephard, and Doornik (1999). The graphics are produced by the accompanying program GiveWin.
} 


\section{Table 1: Parameter Estimates}

The table contains the parameter estimates for the models in (7) through (11). AR1 gives the results for model (7)-(9). AR2 gives the results for (8)-(10). CYC gives the result for model (11). The frequency of cycle $i$ is denoted by $\lambda_{i}$, from which the period can be derived, and its (autoregressive) dampening factor is denoted by $\phi_{i}$. We estimate models with (TV) and without (CV) a time-varying loading for the short cyclical component. Time variation is modeled by 3 dummy variables over the ranges 1927-1948, 1949-1975, 1976-1997.

Diagnostics reported are the loglikelihood value $L$, the Durbin-Watson (DW), the Bowman-Shanton normality test $N$ which has a $\chi^{2}(2)$ distribution, a test for heteroskedasticity $H(23)$ which follows an $F(23,23)$ distribution and checks whether the first and last 23 residuals have equal variance, the first and fifteenth order autocorrelation of the prediction errors $r_{1}$ and $r_{15}$, and the Ljung-Box test for serial correlation $Q(15)$ which has a $\chi^{2}(15)$ distribution. The $95 \%$ confidence bands are in brackets. The number of observations is 71. Significance for $\mathrm{N}, H(23)$, and $Q(15)$ at the $10 \%, 5 \%$, and $1 \%$ level is denoted by ${ }^{a},{ }^{b}$, and ${ }^{c}$, respectively.

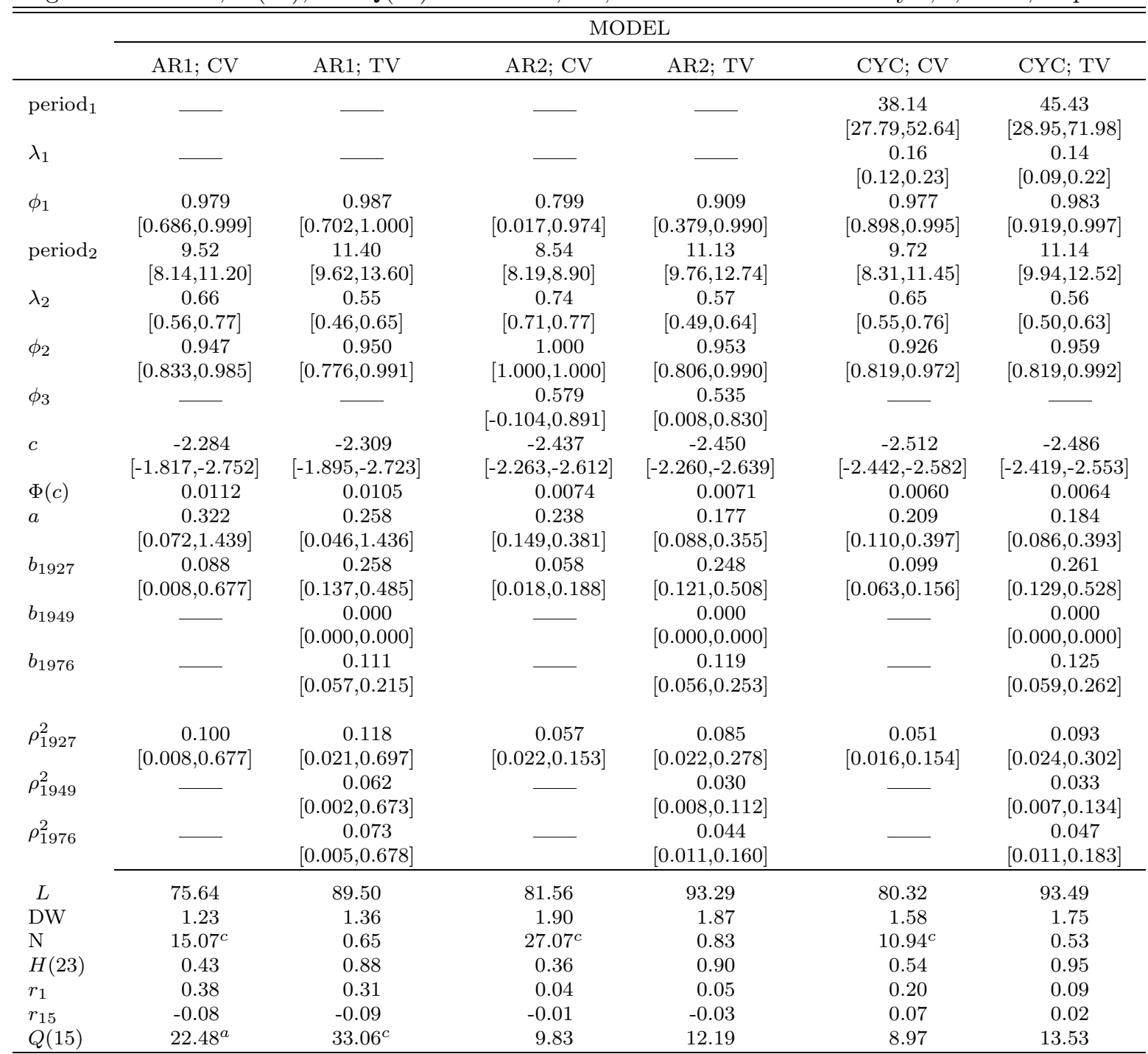

cycle is thus almost nonstationary, similar to the AR(1) component earlier. The short cycle has a reasonable period of 9.7 years. The cyclical component explains about $18 \%$ of the time variation in default rates, which is substantially more than for the previous two models. Note that the unconditional default probability $\Phi(c)$ for the $\operatorname{AR}(2)$ model is the lowest of the models considered. The asset correlation is again estimated at about 5\%. Close inspection of the smoothed component estimates revealed that there is evidence of volatility changes in the cyclical component $\psi_{2 t}$. This is the issue we address next. 


\subsection{Results for time-varying (short) cycle loadings $b_{t}$}

Given the empirical indications (not shown) for a time-varying variance of the short cyclical component, we now allow for time variation in the factor loading $b_{t}$. Given the limited number of data points, we allow for three levels of $b_{t}$. The break dates appear to lie around 1950 and 1975. We vary the break dates between 1947-1952 and 1970-1977, respectively. We choose the set of dates that yields the highest likelihood value. This gives the break dates 1949 and 1976. The closest alternatives in terms of likelihood values all lie in the range 1949-1951 and 1974-1976. We test whether the increase in likelihood value due the addition of two parameters to account for the change in variance is statistically significant. The $p$-value for all models considered is below 0.000008 . Note, however, that this is not a formal test, as the break date is unknown. ${ }^{4}$ To circumvent this issue, we use a pragmatic approach and consider the variation in $p$-values over the range of alternative break points considered. It turns out that the maximum $p$-value over all the different break dates studied is never above 0.0008 for any of the models. From this we draw the conclusion that there is sufficient and convincing evidence for the existence of two breaks in variance. Interestingly, when estimating the $\mathrm{AR}(2)$ model unrestrictedly, it yields complex versus real roots in about $50 \%$ of the cases if we vary the break dates. This indicates that it is difficult to distinguish the long cycle from long non-cyclical movements empirically given the data at hand. The empirical congruence of the two models with respect to the data have led us to use both models in the credit risk analyses rather than arbitrarily dropping one of them. This also allows us to study whether two econometrically similar models also yield similar financial implications.

The parameter estimates can be found in Table 1 in the columns labeled TV. We also present the smoothed component estimates $c+\sqrt{1+a^{2}+b_{t}^{2}}+a \mu_{t}$ and $b_{t} \psi_{2 t}$ with corresponding $95 \%$ confidence bands in Figure 2.

Interestingly, we learn from Figure 2 that there is a close agreement between the different models. In the right-hand plots, there is a pronounced short cyclical effect before 1949, and somewhat less after 1976. In the intermediate period, no short cyclical component is identified. This corroborates the empirical pattern mentioned for the models with fixed $b_{t} \equiv b$. The slowly varying components in the left-hand panels also reveal very similar patterns: there is a slow variation in default rates over longer periods, but periodic departures (or cyclical movements) from these 'benchmark' default rates can be substantial.

The estimation results in Table 1, however, present some subtle differences. The parameters $\operatorname{period}_{2}, \lambda_{2}$, and $\phi_{2}$ of the short cyclical component are almost identical. Only the confidence intervals are slightly wider for the $\mathrm{AR}(1)$ plus cycle specification. The dominant $\mathrm{AR}$ root $\phi_{1}$ for the slowly varying component is similar for the $\mathrm{AR}(1)$ and double cycle model, 0.987 and 0.983 , respectively. For the $\operatorname{AR}(2)$, however, the dominant root of 0.909 is substantially smaller. Consequently, the half-life is only about one third of that for the other two models. Moreover, the parameter $\phi_{1}$ for the $\operatorname{AR}(2)$ specification has a much wider confidence interval. This is in line with well-known results for this class of models: direct modeling of unobserved components in terms of primitive $\mathrm{AR}(1)$ and cyclical components allows for richer dynamics in a more parsimonious way than unrestricted higher order AR specifications, see Harvey (1989). The loading $a$ of the slowly varying component also differs between the AR(1) on the one hand, and the richer $\operatorname{AR}(2)$ and double cycle specifications. The wide confidence band for $a$ as found in the $\operatorname{AR}(1)$ specification with constant $b_{t}$ remains. The unconditional default probabilities $\Phi(c)$ for all models are about the same as for the specification with constant short cycle loading

\footnotetext{
${ }^{4}$ In principle, one should use a supremum likelihood ratio test, compare Vogelsang (1997) in a different context. Deriving and studying such a formal testing procedure is, however, beyond the scope of the current paper.
} 

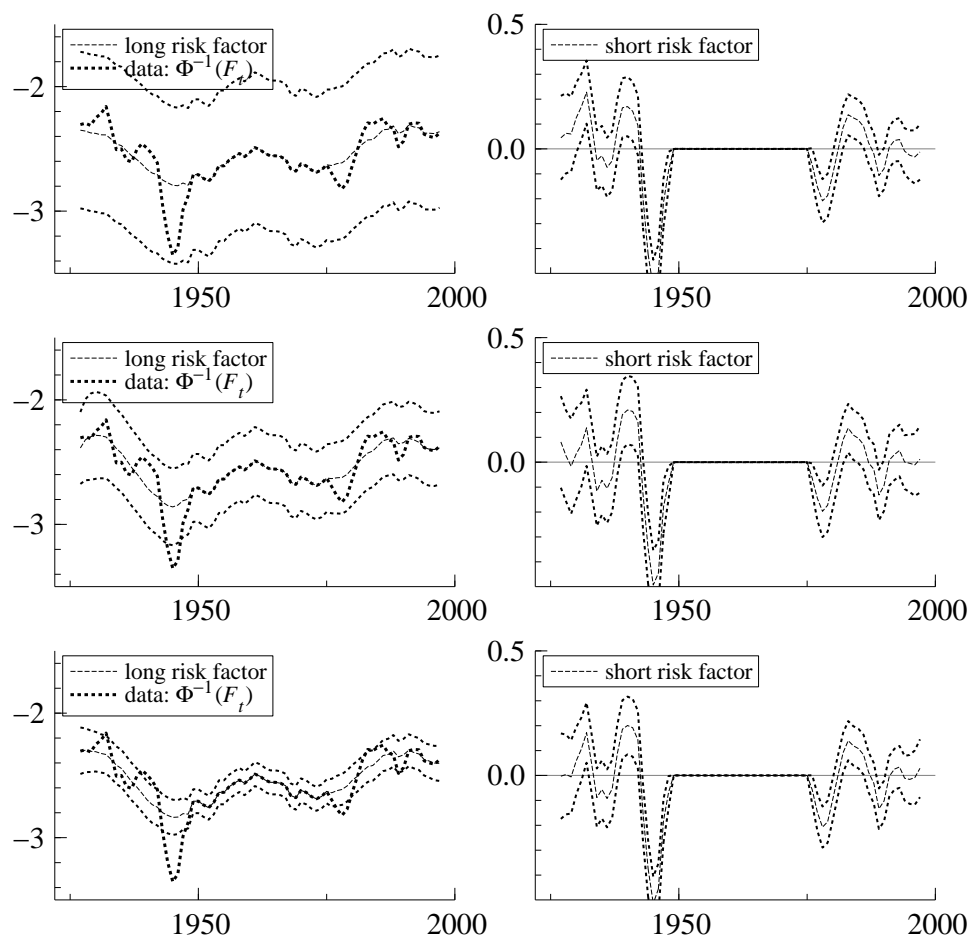

Figure 2: Dynamic Model Fit

The figure presents the smoothed component estimates for the slowly varying and short cyclical components of three alternative dynamic models for the transformed failure rate $\Phi^{-1}\left(F_{t}\right)$, with $F_{t}$ the original failure rate. The top two graphs give the result for the $\operatorname{AR}(1)$ plus cycle, where the right-hand hand plot gives $b_{t} \psi_{2 t}$, and the left-hand plot gives $y_{t}=c \sqrt{1+a^{2}+b_{t}^{2}}+a \mu_{t}$, with $\mu_{t}$ and $\psi_{2 t}$ satisfying (7) and (8). The middle two graphs are for the $\operatorname{AR}(2)$ plus cycle model, where the term $\mu_{t}$ now has to be interpreted as an $\operatorname{AR}(2)$ process with real roots. The bottom two graphs give the result for the double cycle model, where $\mu_{t}$ is replaced by $\psi_{1 t}$, a cyclical component with similar specification as in (8), but longer period. All results take $b_{t}$ to be piecewise constant over the ranges 1927-1948, 1949-1975, 1976-1997.

$b$, see the previous subsection. They range from $0.65 \%$ for the double cycle model to $1.05 \%$ for the $\mathrm{AR}(1)$ plus cycle model.

The time varying factor loadings $b_{t}$ are very similar across all three models. There is a high $b_{t}$ of about $25 \%$ in the years up to 1949 . Note that due to the unit variance parameterization of the short cyclical component in (8), $b_{t}^{2}$ can be interpreted as the variance of the (short) cyclical component in the transformed default rates $y_{t}$. For the $\mathrm{AR}(1)$ plus cycle model, this means that about $50 \%$ (or $b_{t}^{2} /\left(a^{2}+b_{t}^{2}\right)$ ) of the time variation in default rates during $1927-$ 1948 can be attributed to cyclical effects. For the other two models, the figure is substantially higher, namely around $66 \%$. The remaining $50 \%$ and $34 \%$, respectively, can be attributed to more gradual long-term developments in default rates. After the first oil crisis, the standard deviation of the short cycle in $y_{t}$ more than halves with respect to 1927-1949. This can be seen from the value of $b_{1976}$, which is about $11 \%$ or $12 \%$. Consequently, the percentage of variation in default rates over time explained by the cyclical factor decreases dramatically from $50 \%$ and $66 \%$ for the $\mathrm{AR}(1)$ and the other two models to about $16 \%$ and $31 \%$, respectively. The remainder is explained by gradual longer term developments. If we compare these results to those of the previous subsection, we see that by not discriminating between different regimes over time, one may seriously underestimate (pre-1949 and post-1976) as well as overestimate (1950-1978) the impact of short cyclical movements on the total variation in default rates at any time. 


\section{Capital requirements}

So far, we have concentrated on the empirical behavior of failure rates. As argued in the introduction, however, the dynamic behavior of default rates is especially relevant for determining capital requirements in a multi-period setting. There are at least two reasons why this is an important topic. First, both regulators and financial institutions themselves are not only interested in a static, one-year horizon for bankruptcy. They also take the dynamic aspects of their portfolio into account. Second, risk sensitive capital requirements as proposed in the New Capital Accord, Basle Committee on Bank Supervision (2001), may result in an amplification of macroeconomic cycles and in an untimely provisioning for loan losses, see Laeven and Majnoni (2002). To address these issues, we set up a simulation experiment in the present section. The motivation for this experiment is to test whether dynamic models such as estimated in Section 4 result in a more timely accumulation of capital when needed.

Let us consider the following simple balance sheet development of a hypothetical bank:

$$
\begin{aligned}
V_{t} & =\left(1-p_{t}\right) V_{t-1}, \\
D_{t} & =(1+h) D_{t-1}-\left(1-p_{t}\right) g V_{t-1}-p_{t} \delta V_{t-1}, \\
E_{t} & =V_{t}-D_{t},
\end{aligned}
$$

where $D_{t}, E_{t}$, and $V_{t}$ denote the bank's debt level, equity level, and the value of its asset portfolio at time $t$, respectively, and with $D_{0}, E_{0}$, and $V_{0}$ exogenous. Furthermore, $g$ is the (fixed) coupon on the asset portfolio, $h$ denotes the bank's funding rate, $\delta$ is the recovery rate as a fraction of $V_{t}$, and $p_{t}$ is the default rate during period $t$ for the loan portfolio. The balance sheet can be interpreted as follows. Starting with a portfolio of nominal value $V_{t}$ financed by debt $D_{t}$ and equity $E_{t}$, a fraction $p_{t}$ of the portfolio defaults. This results in recovery payments worth $p_{t} \delta V_{t}$. The non-defaulted part of the portfolio $\left(1-p_{t}\right) V_{t}$ pays coupons worth $\left(1-p_{t}\right) g V_{t}$. We assume that coupon and recovery payments are deducted from debt. Alternatively, we could assume that they are reinvested in assets with similar cash flow and risk characteristics as the original portfolio. Adding the funding costs $h D_{t}$, we arrive at (16) and (17). Equation (18) then follows directly.

The default rate $p_{t}$ is modeled as

$$
p_{t}=\Phi\left(y_{t}\right),
$$

with $y_{t}$ given by one of the time series models discussed in Section 4. Given our empirical results, we concentrate on the $\mathrm{AR}(2)$ plus cycle and the double cycle model with time varying variances. Note that (19) only contains one stochastic variable, the transformed default rate $y_{t}=\Phi^{-1}\left(F_{t}\right)$. By using (19), we are abstracting from the effect of idiosyncratic risk factors. This is a reasonable approximation to actual losses if the portfolio is sufficiently fine-grained.

The recovery rate $\delta$ is set to $50 \%$ in our experiment. The funding cost is fixed at $4.5 \%$, while the coupon varies between $4.8 \%$ and $5.4 \%$. Unreported additional simulations reveal that alternative parameter values result in qualitatively similar findings to the ones we will report below. Note that the recovery rate of $50 \%$ leads to an expected loss of $p_{t} / 2$. The default probability in our simulations hovers around 60 or 75 basis points, implying that the difference between coupon and funding costs is approximately equal to the expected loss. The bank's net margin (net of the expected loss) is thus close to zero. By concentrating on this region, we are able to highlight some of the main features of capital requirement determination in a multi-period setting.

To perform our test on the relation between dynamic default models, capital requirements, and pro-cyclicality, we use an unconditional and a conditional approach. In our conditional approach, we proceed as follows. First, we construct for every year in the sample a perfect 
foresight capital requirement (PFCR) corresponding to a one up to ten year planning horizon. The PFCR in a specific year equals the amount of initial capital $E_{0}$ needed to get the bank safely through the planning period, i.e., without ever having a negative equity value during the planning period. From a regulatory perspective, this can be thought of as an absolute minimum capital requirement (for a specific planning horizon) needed if one would observe future default rates developments with certainty. Next, we consider multi-period capital requirements for three different approaches. The first two are based on the $\mathrm{AR}(2)$ plus cycle model and the double cycle model of Section 4, respectively. For every year in the sample, we use the model's parameter estimates to obtain filtered estimates of the slowly varying and the cyclical component. These filtered states are the estimates of $\mu_{t}, \psi_{1 t}$, and $\psi_{2 t}$ given all data up to and including time $t$. Using the filtered states as initial conditions and the estimated dynamic model, we simulate 10,000 paths of 10 year default rate developments. Using the simulations, we perform a grid search to determine the initial capital level $E_{0}$ needed to get the bank safely through the next one up to ten years with a $99.95 \%$ confidence level. The $99.95 \%$ confidence level is interpreted here in the sense that the bank has a fixed annual default hazard rate of 5 basis points. The computations are performed for both dynamic models and for every year in the sample.

Our approach with fixed default threshold $c$ in (13) can be interpreted as rating through the cycle, see Carey and Hrycay (2001). Major rating agencies claim this is in broad agreement with their rating objectives. Banks, however, are arguably claimed to be more aggressive in their rating activity in the sense that business cycle movements have a direct impact on the estimated probabilities of default of borrowers. This effect may be amplified if banks estimate probabilities of default based on recent data only, e.g., five years as a minimum required in the proposed New Capital Accord, Basle Committee on Bank Supervision (2001). As mentioned earlier, active re-rating based on recent observed default figures combined with risk sensitive capital requirements may lead to pro-cyclicality. To mimic this re-rating strategy, we use the following approach. For every year in the sample, a 5 year window of current and past data is used to estimate default probability. The default probability over the future planning period is set to the historical average over the 5 year data window. To estimate the asset correlation $\rho_{t}$ over the planning period, the default rate variability over the past 10 years is used, see Gordy (2000) and Finger (1999) for formulations of the estimator. This is tantamount to calibrating the model in terms of default rather than asset correlations, which is the appropriate thing to do for this class of models, see Frey and McNeil (2001,2002). Note that our present stylized re-rating strategy only catches part of the practice in the banking industry. Banks may also use extraneous information, such as balance sheet data or equity volatility as inputs to their credit scoring methods, see Carey and Hrycay (2001). This is, however, difficult to implement in our study given the current aggregate data level and the limited number of observations.

Using the estimated default correlation and probability of default, future default probabilities are simulated under the assumption of an i.i.d. systematic risk factor $f_{t}$. The default figures can be used to work through the bank's balance sheet, compute the required levels of initial capital, and compare the approach to the dynamic models. Figure 3 presents the required levels of capital for one up to ten year planning periods, conditioned on the filtered states in years 1965, 1980, 1991, and 1997.

We first start with discussing the results for 1965. For low coupons (4.8\%), the differences between the models are clear. Whereas the re-rating approach (top) requires a roughly constant capital level independent of the planning horizon, the dynamic models require higher levels of capital at longer horizons. The effects become even more apparent when conditioning on 1980, which was at the start of the junk bond crisis. Given that at the time the recent past revealed low default figures, the re-rating approach results in very low capital requirements for all coupon levels. Moreover, even for a low coupon of $4.8 \%$, the capital requirement decreases 

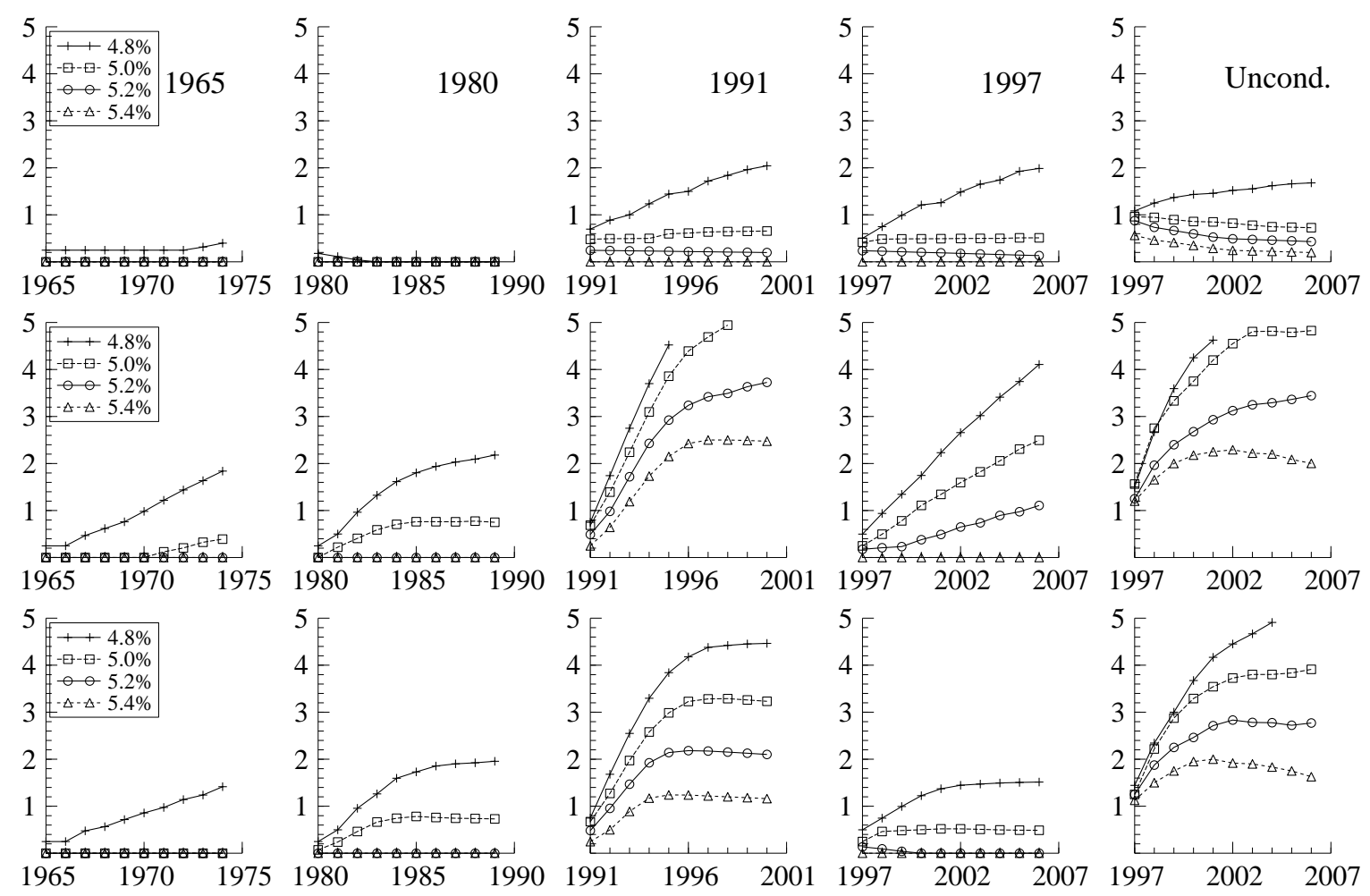

Figure 3: Multi-period capital requirements

The figure contains the capital requirements for a bank to match its pointwise simulated multi-year cumulative default frequency to $1-(99.95 \%)^{t}, t \leq T$, for a planning horizon $T=1, \ldots, 10$. The top line of graphs gives the results for the re-rating strategy where probability of default and default correlation are based on the most recent 5 and 10 year history. The risk factor in this approach is assumed to be i.i.d. The second line of graphs contains the results for the $\operatorname{AR}(2)$ plus cycle model. The bottom line of graphs gives the results for the double cycle model estimated in Section 4. For the middle and lower line of graphs, the initial conditions for the model are the filtered estimates of the states (slowly varying and cyclical unobserved component) in the years 1965, 1980, 1991, and 1997 (column 1, 2, 3, and 4 respectively). These years give an impression of the range of possible patterns. The 5 th column of graphs gives the results for the different models when using an unconditional simulation. In this simulation, the initial conditions are drawn from the unconditional distribution of the states. For the top-right graph, this means that the default probability and correlation were estimated using the complete sample rather than the most recent 5 and 10 years. Each graph contains four curves, which relate to different coupon values on the bank's bond portfolio. The funding costs are fixed at $f=4.5 \%$ in all graphs.

with the planning horizon. This is due to the fact that the re-rating approach is based on an i.i.d. assumption for the systematic risk factor. A depletion of the initial capital buffer by two or more consecutive bad realizations of $f_{t}$ is relatively unlikely under this assumption. Consequently, the 2-year buffer can be set lower because of the projected buffer replenishment by the coupon payment. By contrast, the $\mathrm{AR}(2)$ and double cycle model allow for stickiness in adverse economic conditions through the dynamical structure of the model. As a result, buffer depletion by several bad years in a row is much more likely. The losses produced by this effect cannot be (completely) offset by the coupon payments, such that the initial capital buffer needs to be increased in order to survive. A nice feature is seen for a coupon level of $5.0 \%$. This curve is slightly hump-shaped. The initial increases in the capital level over longer horizons has the same intuition as before. Over even longer horizons, however, every downward cycle must ultimately end. When the cycle stabilizes, the coupon payments on average (more 
than) compensate for the default losses. As a result, the capital requirement at longer horizons stabilizes or even slightly decreases. Note that the empirical similarity between the $\operatorname{AR}(2)$ and double cycle model found in Section 4 result in similar capital requirements when conditioned on the states in 1965 and 1980.

A different picture emerges when conditioning on 1991 or 1997. First note that even the rerating approach indicates that higher capital levels are needed than in 1965 or 1980. Moreover, the increasing pattern for a coupon of $4.8 \%$ clearly indicate that this coupon is too low to prevent a continuous buffer erosion by defaults. A second feature evident in the figure is the large capital levels for the $\mathrm{AR}(2)$ model vis-à-vis the double cycle model, despite the empirical similarity of the two. The main driver of this difference is the downward swing in the long cycle for the double cycle model, which compensates for the movements in the short cycle. A similar compensation is lacking for the AR(2) model, such that higher capital levels are required. For all experiments it is clear that a key role is played by the bank's net margin, i.e., the coupon minus funding costs minus expected loss. If this margin is low (e.g., for a coupon of $4.8 \%$ ), the capital buffer increases. For higher margins, capital requirements level off sooner. For even higher coupon levels, the requirement may start decreasing again for long enough horizons, resulting in the hump-shaped pattern mentioned earlier. This is due to the deterministic positive drift in the buffer level over time due to the (high) coupon payments.

The final column of graphs in Figure 3 contains the results for the unconditional approach. For the dynamic models, the estimated default threshold is based on the complete sample. The initial conditions for the slowly varying and cyclical unobserved component are not fixed at their filtered estimates, but are drawn from the unconditional distribution. Given for example (8), this implies that the first value of $\left(\psi_{2 t}, \psi_{2 t}^{*}\right)$ is drawn from the standard bivariate normal. For the top-right hand graph, the estimates of default probability and asset correlation are based on the complete data rather than the most recent 5 and 10 years. This results in a default probability of 62 basis points and an asset correlation $\rho^{2}=3.31 \%$. The unconditional capital levels are significantly higher than the ones presented earlier. Even for coupons of $5.4 \%$, non-trivial buffer levels emerge. For the top-right graph, note that the bank's net margin is $4.8-4.5-0.62 / 2=-1 \mathrm{bp}<0$. So the coupons do not make up for the expected loss plus funding costs. As a result, the capital is constantly depleted on average over time, such that in order to survive the capital requirement must constantly increase with the planning horizon. The reverse holds for higher coupon levels, which show a clear decreasing pattern. The $\operatorname{AR}(2)$ and double cycle model, by contrast, result in increasing capital levels over short planning horizons. If the coupon is sufficiently high, the earnings drift ultimately takes over and a hump-shaped pattern again emerges.

It must be emphasized that all these simulations are stylized in the sense that in reality banks have the option to issue new equity and to tune the dividend policy in order to strengthen the capital level. These issues are not taken into account here and may be difficult to model in general. However, the simulations so far underline the important effects of stickiness in macroeconomic conditions for default risk and capital buffer formation. Moreover, in our current dynamic setting the bank's net margin appears to be of paramount importance for determining satisfactory levels of capital. This net margin does not appear directly in the new Basle proposals for capital regulation.

\section{Pro-cyclicality}

We now turn to the issue of pro-cyclicality. So far, we have determined what capital requirements are implied by the different modeling approaches. The next important question is 

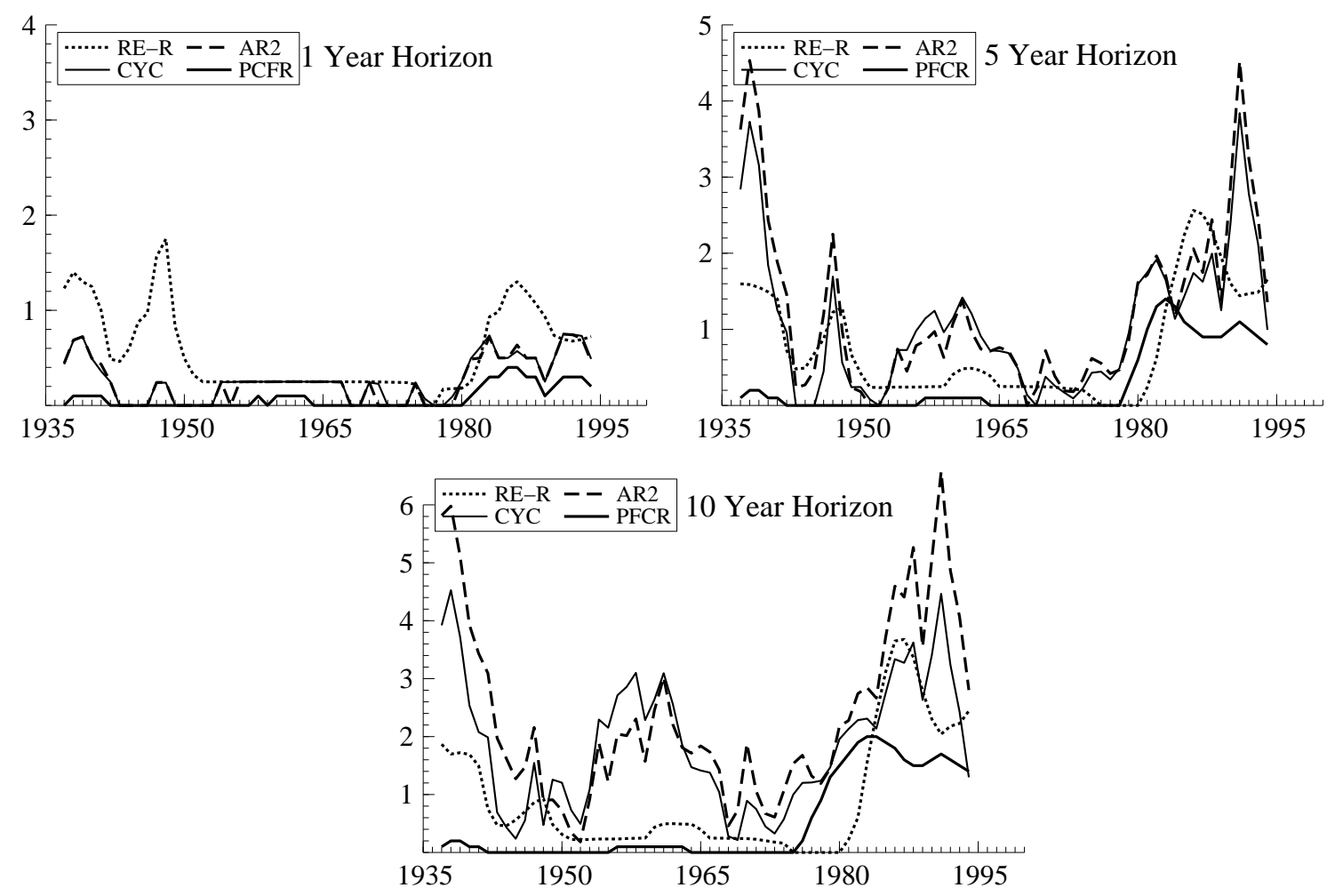

Figure 4: Conditional capital requirements

The figure contains the capital requirements for a bank to match its pointwise simulated multi-year cumulative default frequency to $1-(99.95 \%)^{t}, t \leq T$, for a planning horizon $T$ of 1,5 , or 10 years. Each graph is for a fixed planning horizon and contains four curves: the perfect foresight capital requirement (PFCR), the capital requirements based on a re-rating strategy using recent 5 and 10 years of historical data (RE-R), and the capital requirements following from the $\mathrm{AR}(2)$ plus cycle and double cycle (CYC) model models from Section 4. The curves have been obtained by means of simulation. Each point in a graph contains the initial capital requirement when the initial conditions for the unobserved components are the filtered estimates of the states. The bank's balance sheet evolves according to (16) through (19) with funding cost $f=4.5 \%$, coupon equal to $g=4.8 \%$, and recovery rate $\delta=50 \%$.

whether the model-implied capital levels lag, lead, or coincide with capital levels actually required. The actual level needed is modeled by the PFCR. For three different planning horizons, Figure 4 presents the results.

For a planning horizon of one year, the re-rating approach gives somewhat higher and more volatile capital requirements. All methods give one-year capital levels above the PFCR. The junk bond crisis is clearly visible by the rising PFCR and model implied capital levels in 1980 . The picture changes dramatically if we look at a planning horizon of five years. Most of the time, the dynamic models result in higher capital levels than the re-rating approach. There is an exception during 1983-1990 to which we come later. The most striking feature in the figure is of course found around the start of the junk bond crises, which appears the most stressing event in the sample period. The PFCR now starts rising around 1978 rather than 1980 as for the one-year horizon. The re-rating approach, by contrast, produces increases in the capital level only as of 1980. The intuition is clear. Only in 1980 the first indications arrive of the oncoming crises in the form of increased default numbers. These numbers, in turn, increase the estimated future credit risk and lead to higher capital requirements. A reverse effect is found after the crisis. The capital levels keep rising for a few years, even if the worst of the 
crisis is over. This follows from the fact that default figures from the recent past at the time were still very high. The pattern of lagging capital buffer accumulation and decumulation is typical for the pro-cyclicality problem. It is therefore the more interesting to note that the dynamic models estimated in this paper already indicate that capital levels should increase around 1975. This stems from the fact that these models explicitly account for possible future cyclical effects in default figures. This timely warning allows banks and regulators to increase buffers earlier and at a more reasonable pace (compare the steep increase for the re-rating curve from 1980 onwards). We do not claim that the absolute capital levels indicated in the figure are representative of what should be implemented in an actual regulatory framework. But it is clear that models that explicitly account for the dynamic structure of defaults may help in overcoming part of the pro-cyclicality problems by providing early warning mechanisms. Also note that capital decumulation around 1983 is also much more timely for the dynamic models. In this way, capital requirements based on them may also provide a more efficient regulatory framework. If we turn to the last panel in Figure 4 for a horizon of 10 years, the effects are very similar. Only the absolute capital levels for surviving over 10 years are higher than for 5 years.

For completeness, we also look at the pro-cyclicality issue using an unconditional approach. In the unconditional approach, we simulate future default path scenarios by generating from the unconditional distribution rather than conditioning on the filtered states to start the simulations. As a comparison to our dynamic models, we consider two static alternatives. In both of these, $y_{t}$ is an i.i.d. process. Given the mean and standard deviation of the failure rate over $1927-1997$ of $0.617 \%$ and $0.336 \%$, respectively, we recover the static default threshold $c$ and correlation parameter $\rho$. Using the method of moments, note from (2) that the mean default rate equals $\Phi(c)$, whereas the variance of the default rate equals $\Phi_{2}\left(c, c ; \rho^{2}\right)-\Phi(c)^{2}$, with $\Phi_{2}\left(\cdot, \cdot ; \rho^{2}\right)$ the bivariate normal distribution function for two correlated standard normals with correlation $\rho^{2}$. This leads to a default threshold of -2.50 and a correlation parameter of $\rho^{2}=3.31 \%$. Note that this is slightly lower than the correlations presented in Table 1. Our second model estimates the default threshold $c$ and asset correlation $\rho^{2}$ by means of maximum likelihood, using the fact that the mean and variance of $y_{t}$ are $c / \sqrt{1-\rho^{2}}$ and $\rho^{2} /\left(1-\rho^{2}\right)$, respectively. The resulting values for $c$ and $\rho^{2}$ are $c=-2.56$ and $\rho^{2}=5.18 \%$.

The main conclusion to be drawn from Figure 5 is that dynamic models combined with unconditional capital requirements lead to prudent capital levels given the historical scenario for planning horizons of more than one year. The re-rating based on recent data is also prudent over most of the range. Especially in the early 80s, however, capital levels would have fallen short of the levels needed in a multi-year perspective. This might be undesirable from a regulatory perspective. Finally note that one has to be cautious in labeling any of the above approaches too prudent. Given the limited data span of 70 years and the small default probability of 5 basis points or once every 2000 years, we would indeed not expect the historical scenario to come anywhere near the capital requirement. In this sense, the dynamic models also do a much better job in the unconditional setting.

\section{Conclusions}

In this paper we have analyzed US corporate failure rates over the period 1927-1997 using time series models based on unobserved components. We identify long term movements and cyclical effects in failure rates. The cyclical effects correspond to so called credit cycles and have similar periods (9.5 to 11.5 years) as business cycles. The main advantage of our approach, however, compared to models that incorporate observable business cycle models, is that our 

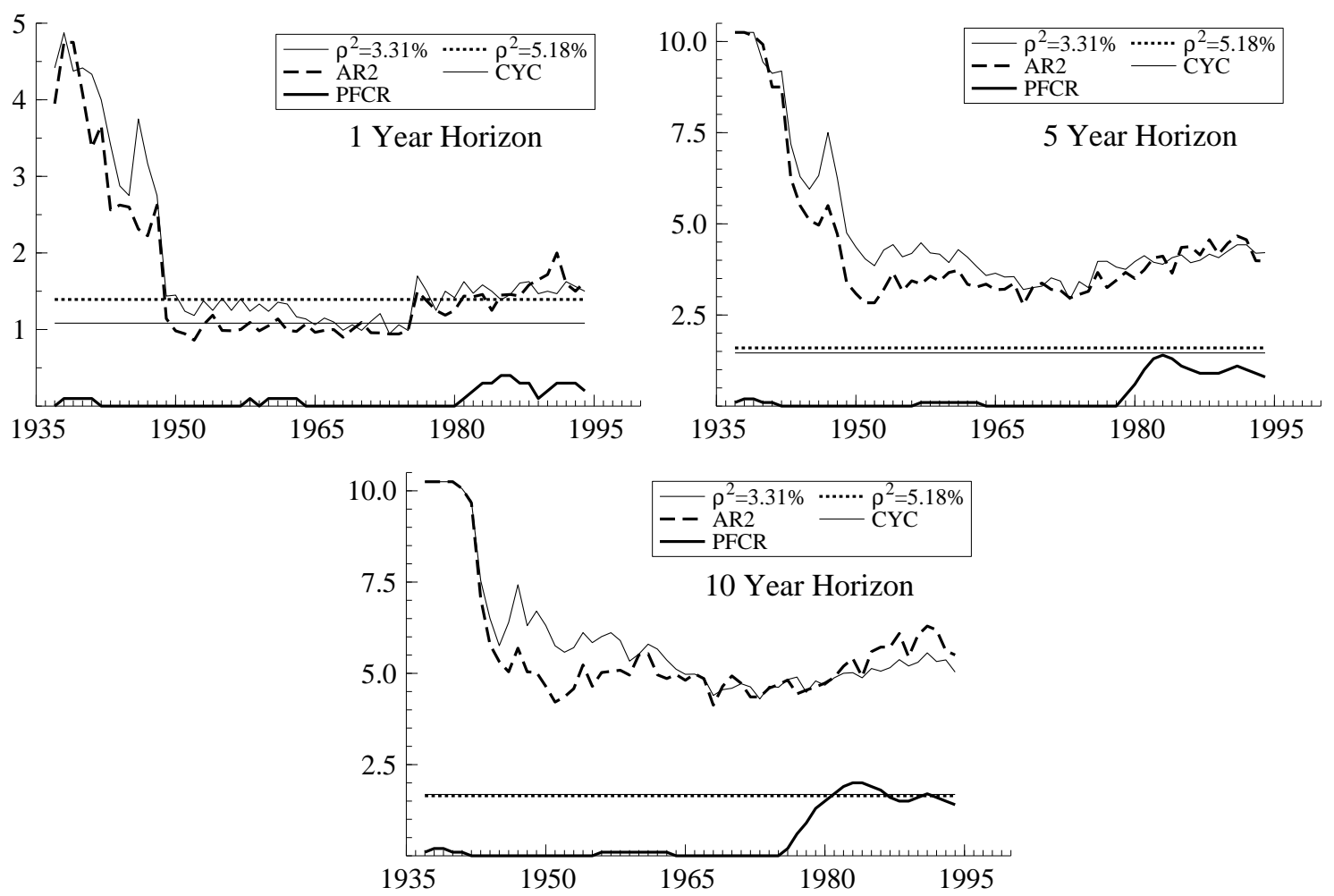

Figure 5: Unconditional capital requirements

The figure contains the capital requirements for a bank to match its pointwise simulated multi-year cumulative default frequency to $1-(99.95 \%)^{t}, t \leq T$, for a planning horizon $T$ of 1,5 , or 10 years. Each graph is for a fixed planning horizon and contains five capital requirement curves: an i.i.d. model with default probability of $62 \mathrm{bp}$ and default correlation $3.31 \%$, the same model but with default probability $52 \mathrm{bp}$ and asset correlation $5.18 \%$, the $\mathrm{AR}(2)$ plus cycle and double cycle (CYC) model models from Section 4, and the perfect foresight capital requirement (PFCR). The curves have been obtained by means of simulation. Each point in a graph contains the initial capital requirement when the initial default threshold $c$ in (9) or (11) is the filtered estimate of the (level) state, while the other components are drawn from their unconditional distribution. The bank's balance sheet evolves according to (16) through (19) with funding cost $f=4.5 \%$, coupon equal to $g=4.8 \%$, and recovery rate $\delta=50 \%$.

model provides an unified simulation framework for dynamic credit risk analyses. Using our integrated modeling approach, estimation is also more efficient than in the commonly used twostep procedure, which first estimate the relation between macroeconomic variables and default rates, and then separately model the dynamic behavior of the macroeconomic variables.

We investigated several model specifications and found that there are two competing models that best fit the data. Both models have second order long term dynamics and a short cycle with a period of about 8.5 to 11 years. The long term dynamics can either be described by an autoregressive component, or by a long cyclical component with a period of about 40 years. Interestingly, we showed significant empirical evidence for structural breaks in the variance of the short cyclical component. Time variation in this variance implies time-varying asset and default correlations. This adds another complexity to the difficulty in estimating these correlations reliably. As Gordy and Heitfield (2002) argues, one needs long time series for such correlations. Here we show, however, that over longer periods non-stationarity in these correlations is empirically very likely. Moreover, the impact of cyclical factors also varies over time. Whereas more than $50 \%$ of the variation in default rates can be attributed to the short 
cyclical component in the pre-war years, this figure collapses to the range $16 \%-30 \%$ from the mid-70s onwards.

Using a simulation experiment for a stylized bank's balance sheet, we also showed that dynamic default rate models may mitigate some of the pro-cyclicality concerns. By an adequate dynamic modeling of default figures and by focusing on an asset/liability perspective for the bank in a multi-year context, model implied capital requirements appear much more in line with what would have be needed historically. In particular, the timing of starting or stopping capital buffer formation appears much more timely than in case one were to use active re-rating strategies based on recent data only. The dynamic approach advocated in the current paper might therefore turn out to be useful in a regulatory framework as well.

Using our simulation set-up, we also showed that the net interest margin, defined as the coupon minus funding costs and minus expected loss, plays a key role in the formation of capital buffers in a multi-year context. Very low net margins lead to increasing capital requirements in the planning horizon. Very high net margins lead to decreasing requirements. There is an intermediate region, where multi-year capital requirements reveal a hump shaped pattern for specific models. The main implication of this for the regulator is that the soundness of capital buffers also depends on the net margin of the financial institution holding the buffer. Though this may complicate the regulatory framework, it seems an indispensable ingredient in a dynamic context. It is also important that regulators pay close attention to issues of conditionality. Capital requirements may be vastly different if one conditions on the current state of the economic dynamics, or not.

\section{References}

Allen, L. and A. Saunders (2002). A survey of cyclical effects in credit risk measurement models. Technical report, Stern School of Business, New York University.

Bangia, A., F. Diebold, A. Kronimus, C. Schagen, and T. Schuermann (2002). Ratings migration and the business cycle, with application to credit portfolio stress testing. Journal of Banking and Finance 26(2-3), $445-474$.

Basle Committee on Bank Supervision (2001, January). The new Basel capital accord. Report, Bank of International Settlements, Basle.

Caouette, J., E. Altman, and P. Narayanan (1998). Managing credit risk. The next great financial challenge. New York: Wiley.

Carey, M. and M. Hrycay (2001). Parameterizing credit risk models with rating data. Journal of Banking and Finance 25, 197-270.

Carpenter, S. B., W. Whitesell, and E. Zakrajšek (2001). Capital requirements, business loans, and business cycles: An empirical analysis of the standardized approach in teh New Basel Capital Accord. Technical report, Board of Governors, Federal Reserve System.

Credit Suisse (1997). CreditRisk ${ }^{+}$. Downloadable: http://www.csfb.com/creditrisk.

Das, S. R., L. Freed, G. Geng, and N. Kapadia (2002). Correlated default risk. Technical report, Santa Clara University.

Doornik, J. A. (2001). Object-Oriented Matrix Programming using Ox 3.0. London: Timberlake Consultants Press.

Duffie, D. and K. Singleton (1999). Modeling the term structures of defaultable bonds. Review of Financial Studies 12, 687-720.

Dun and Bradstreet (1998). Business failure record. a comparative statistical analysis of geographic and industry trends in business failures in the United States. Technical report.

Durbin, J. and S. J. Koopman (2001). Time Series Analysis by State Space Methods. Oxford: Oxford University Press.

Finger, C. (1999, April). Conditional approaches for CreditMetrics portfolio distributions. CreditMetrics Monitor 1, 14-33. 
Finger, C. (2000). A comparison of stochastic default rate models. RiskMetrics Journal 1(2), 49-73.

Frey, R. and A. J. McNeil (2001). Modelling dependent defaults. Technical report, Federal Institute of Technology, ETH, Zurich.

Frey, R. and A. J. McNeil (2002). VaR and expected shortfall in protfolios of dependent credit risks: Conceptual and practical insights. Journal of Banking and Finance 26, 1317-1334.

Gordy, M. (2000). A comparative anatomy of credit risk models. Journal of Banking and Finance 24(1-2), 119-149.

Gordy, M. B. and E. A. Heitfield (2002). Estimating default correlations from short panels of credit rating performance data. mimeo, Federal Reserve Board of Governers.

Gupton, G., C. Finger, and M. Bhatia (1997). CreditMetrics — Technical Document (1st ed.). http://www.riskmetrics.com.

Hamilton, J. (1994). Time Series Analysis. Princeton: Princeton University Press.

Harvey, A. C. (1989). Forecasting, Structural Time Series Models and the Kalman Filter. Cambridge: Cambridge University Press.

Harvey, A. C. and A. Jaeger (1993). Detrending, stylised facts and the business cycle. J. Applied Econometrics $8,231-47$.

Jarrow, R., D. Lando, and S. Turnbull (1997). A Markov model for the term structure of credit risk spreads. Review of Financial Studies 10, 481-523.

Jarrow, R. and S. Turnbull (1995). Pricing derivatives on financial securities subject to credit risk. Journal of Finance (50), 53-85.

Kavvathas, D. (2001). Estimating credit rating transition probabilities for corporate bonds. Job market paper. http://www.ssrn.com/fen.

Koopman, S. J., N. Shephard, and J. A. Doornik (1999). Statistical algorithms for models in state space form using SsfPack 2.2. Econometrics Journal 2, 113-66. http://www.ssfpack.com/.

Laeven, L. and G. Majnoni (2002). Loan loss provisioning and economic slowdowns: Too much, too late? Technical report, Worldbank.

Lucas, A., P. Klaassen, P. Spreij, and S. Straetmans (2001). An analytic approach to credit risk of large corporate bond and loan portfolios. Journal of Banking and Finance 25, 1635-1664.

Merton, R. (1974). On the pricing of corporate debt: the risk structure of interest rates. Journal of Finance 29, 449-470.

Nickell, P., W. Perraudin, and S. Varotto (2000). Stability of rating transitions. Journal of Banking and Finance 24(1-2), 203-227.

Vogelsang, T. (1997). Wald-type $t$-tests for detecting breaks in the trend function of a dynamic time series. Econometric Theory 13, 818-849. 